\title{
Hemagglutinin-Neuraminidase Gene Sequence-Based Reclassification of Human Parainfluenza Virus 3 Variants
}

\author{
Fahad N. Almajhdi \\ Department of Botany and Microbiology, College of Science, King Saud University, Riyadh, Saudi Arabia
}

\section{Key Words}

Hemagglutinin-neuraminidase protein gene $\cdot$ Human parainfluenza virus 3 - Phylogenetic analysis · Saudi Arabia

\begin{abstract}
The most comprehensive phylogenetic classification of human parainfluenza virus 3 (HPIV-3) was recently developed [PLoS One 2012;7:e43893]. This classification included three distinct clusters (A, B and C) with subdivision of cluster C into four subclusters (C1-4). In the present report, the classification of HPIV-3 was refined by inclusion of 27 overlooked beside newly characterized Saudi variants. The new phylogram was developed and included the same clusters described before, in which cluster $A$ remained unchanged and cluster $B$ contained more recent isolates. The organization of cluster $C$ was altered through inclusion of a new subcluster (C5), subdivision of $\mathrm{C} 1$ into two lineages $\mathrm{C} 1 \mathrm{a}$ and $\mathrm{C} 1 \mathrm{~b}$ and subdivision of $\mathrm{C} 3$ into three lineages $\mathrm{C} 3 \mathrm{a}, \mathrm{C} 3 \mathrm{~b}$ and $\mathrm{C} 3 \mathrm{C}$. The majority of Saudi variants were classified as members of subcluster $\mathrm{C} 1 \mathrm{~b}$, whereas only one variant was placed in each of subclusters $C 2$ and $C 5$. This study illustrates an up-to-date phylogenetic classification of HPIV-3 variants.
\end{abstract}

(c) 2015 S. Karger AG, Basel
(C) 2015 S. Karger AG, Basel

0300-5526/15/0581-0035\$39.50/0
Human parainfluenza viruses (HPIVs) cause a wide range of upper and lower respiratory tract infections (RTIs) in children, immunocompromised adults and the elderly [1]. They account for $62 \%$ of croup cases, one half of laryngitis cases and over one third of all lower RTIs of viral origin in children $[2,3]$. HPIVs are members of the family Paramyxoviridae, order Mononegavirales. Like all mononegaviruses, HPIVs have a single-stranded, monopartite RNA genome of negative polarity and produce enveloped virions with a helical nucleocapsid. On both antigenic and genetic bases, 4 principal HPIV groups (1-4) were described, among which HPIV-1 and -3 are classified as members of the genus Respirovirus and HPIV-2 and -4 as members of the genus Rubulavirus [1, 4]. Epidemics of HPIV-3 infections usually occur in the late spring and summer, whereas peaks of HPIV -1 and -2 infections arise in the late autumn and early winter with 1- or 2-year intervals [5].

Of the 4 viruses, HPIV- 3 is the most common cause of acute lower RTIs in infants and young children; only next to human respiratory syncytial virus [6]. In a retrospective study that analyzed the prevalence of HPIVs in the UK between 1975 and 1997, HPIV-3 accounted for 70.8\% of all reported cases [5]. Pediatric hospitalization due to HPIV-3 infection in the USA was estimated to approach

\section{KARGER 125}

E-Mail karger@karger.com www.karger.com/int
Fahad N. Almajhdi

Department of Botany and Microbiology, College of Science, King Saud University PO Box 2455

Riyadh 11451 (Saudi Arabia)

E-Mail majhdi@ksu.edu.sa 
18,000 cases annually [1]. The virus has also been incremented as a cause of nosocomial outbreaks in newborn nurseries [7] and bone marrow transplantation units [8, 9]. In Saudi Arabia, the health and economic burden of HPIV-3 infections is largely undetermined. Only few reports have identified the virus as a potential cause of infant hospitalization in Abha, Qassim and Riyadh districts of the kingdom [10-14].

The molecular epidemiology and circulation pattern of HPIV-3 variants have received little concern in the literature. Few studies have described an analysis of sequence variation and the phylogenetic relationship of different variants on geographical and temporal basis. The hemagglutinin-neuraminidase $(\mathrm{HN})$ gene was recommended as a primary target for phylogenetic analysis of HPIV-3 due to its higher antigenic and sequence variability [11]. The earliest effort in this regard utilized the nucleotide sequence of the complete fusion gene to separate 15 HPIV-3 variants isolated between 1959 and 1987 in North America into two lineages [15]. Another study separated 32 variants, recovered from a nosocomial outbreak in pediatric oncohematology unit between September 2007 and January 2008, into 5 clusters according to sequence analysis of two variable regions in the $\mathrm{HN}$ gene (nt 1-569 and nt 762-1239) [16].

In a previous report of our laboratory, the entire sequence of $\mathrm{HN}$ gene was utilized to study the phylogenetic relationship between different HPIV-3 variants circulating globally [11]. A distinguished Asian clade was identified in this study and was divided into two subclades: A1 that contained Chinese and Japanese variants and A2 that included Indian and Saudi variants. Soon after, this phylogram was established after analysis of 42 new Chinese variants suggesting the existence of three distinct clusters (A, B and C) with subdivision of cluster $\mathrm{C}$ into subclusters (C1-4) [17]. A significant number of sequences including 4 Saudi variants were not considered in both analyses. In this report, all complete HN gene sequences available to date in GenBank were considered for phylogenetic analysis. Therefore, the study aimed to improve our understanding on sequence variation and build up more reasonable phylogenetic relationship between HPIV-3 variants.

Ten HPIV-3 clinical isolates, identified by nested RTPCR [11], were included in this study. The isolates were recovered from nasopharyngeal aspirate samples collected from hospitalized children with acute respiratory tract illness during 2008-2009 in Riyadh, Saudi Arabia. Isolation of viruses was performed in human epidermoid carcinoma cells, and the virus identity was con- firmed using immunofluorescent and neutralization assays [18]. Two representative variants Riyadh 11/2008 and Riyadh 149/2009 were previously utilized in sequence and phylogenetic analysis [11]. The rest of the Saudi variants $(n=8)$ is the subject of genetic analysis in this study. The variants were utilized in two forms to ensure consistency of the results: (a) aliquots of the original material, (b) clarified supernatant fluid from the first passage in cell culture.

The viral RNA was extracted from the preparations of clinical isolates using QIAamp viral RNA extraction kit (Qiagen, Hilden, Germany) according to the manufacturer's instructions. Three overlapping fragments that span the entire sequence of HN gene were amplified using FideliTaq RT-PCR kit (GE Healthcare, Chalfont St. Giles, UK) and the following primer sets: HPIV3-HN1 (sense: 5'-CGAGATGGAATACTGGAAGC-3', antisense: $\quad 5^{\prime}$-ATCAAGTACAATATCTTCTATGCC-3'), HPIV3-HN2 (sense: 5'-CTGTAAACTCAGACTTGTT ACCTG-3' , antisense: $5^{\prime}$-GCTGTTGAGTAAGTTA TG ACTGG-3'), and HPIV3-HN3 (sense: $5^{\prime}$-CATAATGT GCTATCAAGACCAGG-3' ${ }^{\prime}$, antisense: $5^{\prime}$-CTGATTGC TGATTACTTATCATATACTTG- $3^{\prime}$ ). The amplification products were agarose gel purified using Illustra ${ }^{\mathrm{TM}}$ Gel Band Purification Kit (GE Healthcare) and were sequenced on both strands (GATC Biotech, Cologne, Germany). Sequences were aligned together and assembled using Bioedit program, version 7.2.5 (Ibis Biosciences, Carlsbad, Calif., USA). The nucleotide and predicted amino acid sequences of Saudi HPIV-3 variants analyzed in this study are available from GenBank under the accession numbers HM460886, HM460887, JX131646, JX131647, JX131648, and JX131649.

The entire HN sequence of Saudi HPIV-3 variants was aligned with 53 corresponding sequences available in GenBank (table 1) using Clustal W algorithm of MegAlign program, Lasergene software (DNASTAR, Madison, Wisc., USA). The phylogenetic tree was constructed using the neighborhood joining method of Molecular Evolutionary Genetic Analyses (MEGA) software, version 5.1 [19]. Bootstrapping of 1,000 replicates was calculated to ensure the accuracy of the tree topology. Values that exceed $50 \%$ were indicated at the branch knobs. Only 6 Saudi variants with unique sequences (including Riyadh 11/2008 and Riyadh 149/2009) were utilized for tree construction.

The need for classification of HPIV-3 variants into distinct clusters and/or genotypes was not evident until recently. The number of available sequences in Genbank and relevant databases was very limited during the 20th
36

Intervirology 2015;58:35-40 DOI: $10.1159 / 000369208$
Almajhdi 
century, and all of these sequences were recovered from North America and Australia. The single trial of HPIV-3 classification in this era only generated two lineages of North American variants [15]. By accumulation of HPIV3 sequences in the databases during the last decade, scientists became able to generate more reliable and comprehensive classification structures. During 2012, two phylograms were constructed based on the complete $\mathrm{HN}$ gene sequence; the first suggested an Asian clade [11], while the other proposed the classification of HPIV-3 variants into three clusters and several subclusters [17]. However, both studies overlooked the full genome sequences that essentially include a complete version of $\mathrm{HN}$ gene sequence within. These sequences represent 27 HPIV-3 variants isolated between 2004 and 2011 from different countries across the globe including Argentina, Australia, France, Mexico, South Africa, USA and Zambia.

In this study, all the available full-length $\mathrm{HN}$ gene sequences, either solely or as a portion of full genome sequence, were utilized for generation of an up-to-date phylogenetic classification. Furthermore, 8 newly characterized Saudi variants were sequenced, and variants that possess a unique $\mathrm{HN}$ gene sequence $(\mathrm{no}=4)$ were included in the phylogram. To diminish the complexity of the phylogram, only 9 representative Chinese variants were presented (table 1; fig. 1). A careful phylogenetic analysis was performed to confirm that the selected variants are standing for all Chinese strains. Similar to the study of Mao et al. [17], the current phylogenetic analysis showed that HPIV-3 variants can be divided into three main clusters (A, B and $\mathrm{C}$ ) with a minimal nucleotide divergence of $4.5 \%$ between clusters and an average divergence of $1-2.7 \%$ within clusters. Cluster A was identical in both studies and contained only 2 prototype variants isolated from USA and Australia in 1954 and 1974, respectively. In contrast, cluster B that merely included isolates of the last century in the study of Mao et al. [17] was shown to include many recent isolates from Australia, USA and South Africa in this analysis. Cluster $\mathrm{C}$ remains the most dynamic and widespread group that includes the majority of variants recovered during the 21 st century (fig. 1; table 2).

Members of cluster $\mathrm{C}$ were further subdivided into 5 subclusters designated as $\mathrm{C} 1-5$, with minimal nucleotide divergence of $1.9-3.1 \%$ (mean $2.4-3.6 \%$ ) between subclusters. The bootstrap values ranged from 86 to $100 \%$ excluding the subcluster $\mathrm{C} 4$ that included only one variant from India (IND-DEL322-06) and was not supported by a significant bootstrap value. Comparative alignment of cluster $\mathrm{C}$ lineages generated in this study and that of
Table 1. List of HPIV-3 strains included in phylogenetic analysis

\begin{tabular}{|c|c|c|c|}
\hline Strain & $\begin{array}{l}\text { Accession } \\
\text { No. }\end{array}$ & Country/year & $\begin{array}{l}\text { Included } \\
\text { in previous } \\
\text { phylograms }\end{array}$ \\
\hline ARG-10068-04 & KF530245 & Argentina/2004 & No \\
\hline ARG-15318-07 & KF530257 & Argentina/2007 & No \\
\hline AUS-1-07 & KF530226 & Australia/2007 & No \\
\hline AUS-3-07 & KF530243 & Australia/2007 & No \\
\hline AUS-04 & AY283063 & Australia/2004 & No \\
\hline AUS-5-07 & KF530251 & Australia/2007 & No \\
\hline AUS-6-07 & KF530249 & Australia/2007 & No \\
\hline AUS-7-07 & KF530230 & Australia/2007 & No \\
\hline AUS-9-07 & KF530225 & Australia/2007 & No \\
\hline AUS-124854-74 & M18760 & Australia/1974 & Yes \\
\hline CAN-14702 & EU424062 & Canada/n.a. & Yes \\
\hline CAN-Toronto & $\mathrm{Z} 26523$ & Canada/n.a. & Yes \\
\hline CHN-LZ22-03 & FJ455842 & China/2003 & Yes \\
\hline CHN-BJ009-08 & GU732134 & China/2008 & Yes \\
\hline CHN-BJ156-09 & GU732145 & China/2009 & Yes \\
\hline CHN-BJ232-09 & GU732149 & China/2009 & Yes \\
\hline CHN-BJ262-09 & GU732154 & China/2009 & Yes \\
\hline CHN-BJ306-09 & GU732160 & China/2009 & Yes \\
\hline CHN-BJ327-09 & GU732167 & China/2009 & Yes \\
\hline CHN-BJ341-09 & GU732171 & China/2009 & Yes \\
\hline CHN-ZhYMgz-01 & EU326526 & China/2001 & Yes \\
\hline FRA-27273076-07 & KF530253 & France/2007 & No \\
\hline FRA-29111069-09 & KF530233 & France/2009 & No \\
\hline FRA-30264021-10 & KF530236 & France/2010 & No \\
\hline IND-DEL88-06 & EU814624 & India/2006 & Yes \\
\hline IND-DEL139-05 & EU814626 & India/2005 & Yes \\
\hline IND-DEL322-06 & EU814623 & India/2006 & Yes \\
\hline IND-DELw32-05 & EU814625 & India/2005 & Yes \\
\hline JAP-HA1 & AB189961 & Japan/n.a. & Yes \\
\hline JPN-KK24 & AB189960 & Japan/n.a. & Yes \\
\hline SA-Riyadh 11-2008 & HM460886 & Saudi Arabia/2008 & Yes \\
\hline SA-Riyadh 45-2008 & JX131646 & Saudi Arabia/2008 & No \\
\hline SA-Riyadh 50-2008 & JX131647 & Saudi Arabia/2008 & No \\
\hline SA-Riyadh 63-2008 & JX131649 & Saudi Arabia/2008 & No \\
\hline SA-Riyadh 119-2009 & JX131648 & Saudi Arabia/2009 & No \\
\hline SA-Riyadh 149-2009 & HM460887 & Saudi Arabia/2009 & Yes \\
\hline MEX-1077-04 & KF687319 & Mexico/2004 & No \\
\hline MEX-1110-04 & KF687321 & Mexico/2004 & No \\
\hline MEX-1526-05 & KF530234 & Mexico/2005 & No \\
\hline MEX-2545-06 & KF530250 & Mexico/2006 & No \\
\hline TEX-545-80 & M18762 & USA/1980 & Yes \\
\hline TEX-9305-82 & M18763 & USA/1982 & Yes \\
\hline TEX-12677-83 & M18764 & USA/1983 & Yes \\
\hline USA-629-10-09 & KF687317 & USA/2009 & No \\
\hline USA-629-D1-09 & KF530241 & USA/2009 & No \\
\hline USA-629-D00687-08 & KF530242 & USA/2008 & No \\
\hline USA-629-D01363-08 & KF530252 & USA/2008 & No \\
\hline USA-629- D01929-07 & KF530256 & USA/2007 & No \\
\hline USA-629-D01959-07 & KF687318 & USA/2007 & No \\
\hline USA-629-D02313-06 & KF530247 & USA/2006 & No \\
\hline USA-10991B-10 & KF530232 & USA/2010 & No \\
\hline USA-C243-57 & JN089924 & USA/1957 & Yes \\
\hline USA-JS & $\mathrm{Z11575}$ & USA/n.a. & Yes \\
\hline WASH-649-79 & M18761 & USA/1979 & Yes \\
\hline WASH-1511-73 & M18759 & USA/1973 & Yes \\
\hline ZAF-3372-10 & KF687340 & South Africa/2010 & No \\
\hline ZAF-6538-09 & KF687346 & South Africa/2009 & No \\
\hline ZAF-1145978-07 & KF687336 & South Africa/2007 & No \\
\hline ZAM-ZLMS-11 & AB736166 & Zambia/2011 & No \\
\hline
\end{tabular}




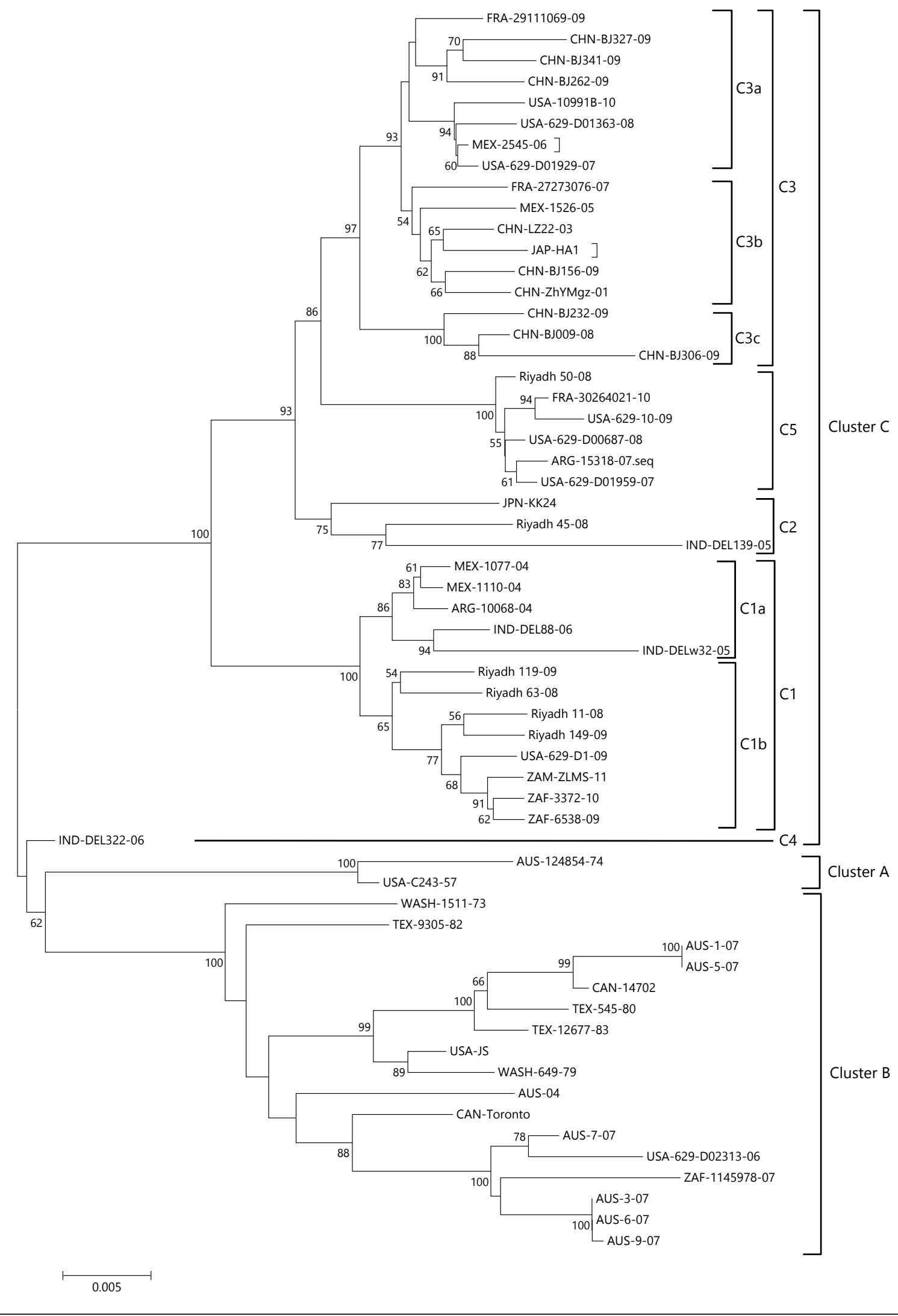

(For legend see next page.) 
Table 2. Alignment of HPIV-3 phylogenetic classes described in this study with that of Mao et al. [17]

\begin{tabular}{ll}
\hline Mao et al. & This study \\
\hline Cluster A & Cluster A \\
Cluster B & Cluster B $^{1}$ \\
Cluster C & Cluster C \\
Subcluster C1 & Subcluster C1 (lineages a, b) \\
Subcluster C2 & Subcluster C2 \\
Subcluster C3 & Subcluster C3 \\
Lineage C3a & Lineages C3a, b \\
Lineage C3b & Lineage C3c \\
Lineage C3c & Subcluster C2 \\
Subcluster C4 & Subcluster C4 \\
& Subcluster C5 \\
\hline
\end{tabular}

${ }^{1}$ Includes additional recent variants from Australia, USA and South Africa.

Mao et al. [17] has demonstrated several important updates: (1) appearance of the novel subcluster C5, which includes variants isolated during the period 2007-2011, (2) subdivision of subcluster $\mathrm{C} 1$ into two lineages $\mathrm{Cla}$ and C1b with a bootstrap value of $100 \%$ and a minimal nucleotide divergence of $1 \%$, (3) placement of the JPN-KK24 variant, the sole member of C3c lineage [17], into subcluster C2, (4) subdivision of subcluster C3 into 3 lineages ( $\mathrm{C} 3 \mathrm{a}, \mathrm{C} 3 \mathrm{~b}$ and $\mathrm{C} 3 \mathrm{c})$ with a bootstrap value ranging from 93 to $100 \%$ and a minimal nucleotide divergence of $0.9 \%$. However, the distribution of variants within C 3 lineages was changed and expanded to contain new variants as compared to the study of Mao et al. [17], where variants of C3a lineage were placed into two separate lineages in this study: C3a and C3b. In addition, C3b lineage was also renamed as $\mathrm{C} 3 \mathrm{c}$ (table 2).

In our previous work of the phylogenetic analysis of HPIV-3 variants, a distinct Asian clade that contained all variants isolated in the Asian continent except INDDEL322-06 variant was proposed [11]. In the current analysis, all members of this clade, beside new Saudi and Chinese variants, were included in cluster C. However,

Fig. 1. Phylogenetic tree of HPIV-3 variants. Complete HN gene sequence of different variants circulating globally was aligned using Clustal W, available within the MegAlign program (DNAstar). The neighborhood-joining method of the MEGA-5 program was used to construct the tree. Accuracy of the tree was confirmed by applying 1,000 bootstrap replicates, and values exceeding $50 \%$ are only denoted at the branches' nodes. Clusters and subclusters are indicated on the right side of the phylogram.

HN Sequence-Based Reclassification of HPIV-3 Variants cluster $\mathrm{C}$ cannot be regarded as an Asian clade since different variants from other continents are also included. Besides, it appears that variants are collected in cluster C on temporal rather than spatial bases (i.e. all the members of cluster C are isolates of 2001-2011 with no geographical restrictions). Further analysis of the subclusters of the Asian clade has revealed a potential correlation with certain $\mathrm{C}$ subclusters. For instance, Chinese and Japanese variants that were included in the $\mathrm{A} 1$ subcluster of the Asian clade are located in the $\mathrm{C} 2$ and $\mathrm{C} 3$ groups, whereas all members of the A2 subcluster are contained in the $\mathrm{C} 1$ group (fig. 1).

A total of 10 Saudi variants were included in this analysis, 6 of which had unique sequences. The majority of variants $(n=4)$ were classified as members of the $\mathrm{Clb}$ sublineage with an overall homology of 98.7-99.3 and 99.5-99.8\% on nucleotide and amino acid levels, respectively. One variant (Riyadh 45/2008) was included in subcluster C2, and another variant (Riyadh 50/2008) was classified as a member of subcluster C5 (fig. 1). These results indicate that HPIV-3 variants of different lineages are co-circulating in Saudi Arabia during the same epidemic seasons, which appears as a consistent criterion observed among different variants circulating in many countries worldwide (e.g. China, India and USA) [7, 17, 20] (fig. 1).

In summary, this study provides an updated and improved version of the phylogenetic classification of HPIV3 that includes all variants available to date according to best of our knowledge. Furthermore, it indicates that variants of different lineages are co-circulated in Saudi Arabia. Further enhancement of the developed phylogram should be done on a regular basis once new HPIV3 sequences are generated. Continuous sequence and phylogenetic analysis of the Saudi variants on geographical and temporal foundations will enable further understanding of the genetic diversity and circulation pattern of HPIV-3 in Saudi Arabia.

\section{Acknowledgement}

This project was supported by NSTIP strategic technologies program number (14-MED809-02) in the Kingdom of Saudi Arabia. The author also thanks Dr. Haitham Amer for his great assistance in genetic analysis and manuscript revision.

\section{Disclosure Statement}

The author declares that he has no conflicts of interest. 


\section{References}

1 Henrickson KJ: Parainfluenza viruses. Clin Microbiol Rev 2003;16:242-264.

$\checkmark 2$ Knott AM, Long CE, Hall CB: Parainfluenza viral infections in pediatric outpatients: seasonal patterns and clinical characteristics. Pediatr Infect Dis J 1994;13:269-273.

-3 Marx A, Torok TJ, Holman RC, Clarke MJ, Anderson LJ: Pediatric hospitalizations for croup (laryngotracheobronchitis): biennial increases associated with human parainfluenza virus 1 epidemics. J Infect Dis 1997;176: 1423-1427.

4 King AM, Adams MJ, Carstens EB, Lefkowitz EJ: Virus Taxonomy and Nomenclature of Viruses. Ninth Report of the International Committee on Taxonomy of Viruses. Amsterdam, Elsevier-Academic Press, 2012.

5 Laurichesse H, Dedman D, Watson JM, Zambon MC: Epidemiological features of parainfluenza virus infections: laboratory surveillance in England and Wales, 1975-1997. Eur J Epidemiol 1999;15:475-484.

-6 Counihan ME, Shay DK, Holman RC, Lowther SA, Anderson LJ: Human parainfluenza virus-associated hospitalizations among children less than five years of age in the United States. Pediatr Infect Dis J 2001;20: 646-653.

7 Karron RA, O'Brien KL, Froehlich JL, Brown VA: Molecular epidemiology of a parainfluenza type 3 virus outbreak on a pediatric ward. J Infect Dis 1993;167:1441-1445.
8 Wasserman R, August CS, Plotkin SA: Viral infections in pediatric bone marrow transplant patients. Pediatr Infect Dis J 1988;7: 109-115.

9 Zambon M, Bull T, Sadler CJ, Goldman JM, Ward KN: Molecular epidemiology of two consecutive outbreaks of parainfluenza 3 in a bone marrow transplant unit. J Clin Microbiol 1998;36:2289-2293.

10 al-Hajjar S, Akhter J, al Jumaah S, Hussain Qadri SM: Respiratory viruses in children attending a major referral centre in Saudi Arabia. Ann Trop Paediatr 1998;18:87-92.

11 Almajhdi FN, Alshaman MS, Amer HM: Molecular characterization and phylogenetic analysis of human parainfluenza virus type 3 isolated from Saudi Arabia. J Med Virol 2012; 84:1304-1311.

12 Alshehri MA, Sadeq A, Quli K: Bronchiolitis in Abha, southwest Saudi Arabia: viral etiology and predictors for hospital admission. West Afr J Med 2005;24:299-304.

13 Bakir TM, Halawani M, Ramia S: Viral aetiology and epidemiology of acute respiratory infections in hospitalized Saudi children. J Trop Pediatr 1998;44:100-103.

14 Meqdam MM, Subaih SH, Thwiny IR: Rapid detection and clinical features of influenza and parainfluenza in infants and young children hospitalized with acute lower respiratory illnesses. J Trop Pediatr 2005;51:160-165.
15 Prinoski K, Cote MJ, Kang CY, Dimock K: Evolution of the fusion protein gene of human parainfluenza virus 3. Virus Res 1992;22: 55-69.

16 Piralla A, Percivalle E, Di Cesare-Merlone A, Locatelli F, Gerna G: Multicluster nosocomial outbreak of parainfluenza virus type 3 infection in a pediatric oncohematology unit: a phylogenetic study. Haematologica 2009;94: 833-839.

17 Mao N, Ji Y, Xie Z, Wang H, An J, Zhang X, Zhang Y, Zhu Z, Cui A, Xu S, Shen K, Liu C, Yang W, Xu W: Human parainfluenza virusassociated respiratory tract infection among children and genetic analysis of HPIV-3 strains in Beijing, China. PLoS One 2012;7: e43893.

18 Maitreyi RS, Broor S, Kabra SK, Ghosh M, Seth P, Dar L, Prasad AK: Rapid detection of respiratory viruses by centrifugation enhanced cultures from children with acute lower respiratory tract infections. J Clin Virol 2000;16:41-47.

19 Tamura K, Peterson D, Peterson N, Stecher G, Nei M, Kumar S: Mega5: molecular evolutionary genetics analysis using maximum likelihood, evolutionary distance, and maximum parsimony methods. Mol Biol Evol 2011;28:2731-2739.

20 Collins KR, Easton AJ: Sequence variation in the haemagglutinin-neuraminidase gene of human parainfluenza virus type 3 isolates in the UK. Epidemiol Infect 1995;114:493-500. 